\title{
Les excitations magnétiques et la diffusion inélastique de neutrons
}

\section{B. Fåk}

ISIS Facility, Rutherford Appleton Laboratory, Chilton Dikcot, Oxon OX11 OQX, U.K.

\section{INTRODUCTION}

La diffusion inélastique des neutrons est une technique qui permet de mesurer les excitations en matière condensée. L'énergie des neutrons froids et thermiques correspond à celle de nombreuses excitations dans ces systèmes et le moment des neutrons permet de parcourir la zone de Brillouin et donc de tracer les relations de dispersion de ces excitations. A cause de son spin, le neutron interagit avec les électrons non appariés et permet donc de mesurer les excitations magnétiques, comme les excitations cristallines ou les ondes de spin. Les bases de la diffusion inélastique sont discutées dans ce cours. Les instruments utilisés pour ce type de mesures, comme les spectromètres à trois axes et à temps de vol sont alors décrit.

\section{NOTIONS DE BASE}

Dans une expérience de diffusion inélastique, l'énergie $\hbar \omega$ transferée de la sonde (le neutron) au système est determinée en mesurant l'énergie du neutron incident $E_{0}$ et diffusé $E_{1}$,

$$
\hbar \omega=E_{0}-E_{1} .
$$

Le vecteur de diffusion $Q$, est obtenu dans le même temps par

$$
\mathrm{Q}=\mathbf{k}_{0}-\mathbf{k}_{1},
$$

où $\mathbf{k}_{0}$ et $\mathbf{k}_{1}$ sont les vecteurs d'ondes des neutrons incidents et diffusés. L'ordre de $\mathbf{k}_{0}$ et $\mathbf{k}_{1}$ sont parfois interchangés dans l'équation (2), en particulier pour la diffusion élastique. L'énergie du neutron est donnée par son énergie cinétique, $E=\hbar^{2} k^{2} / 2 m$, où $m$ est la masse du neutron. Dans certains cas, il est suffisant de connaitre l'amplitude du vecteur de diffusion $Q \equiv|Q|$, qui est obtenu des équations (1-2) par

$$
Q=\sqrt{k_{0}^{2}+k_{1}^{2}-2 k_{0} k_{1} \cos \phi}=k_{0} \sqrt{2-\hbar \omega / E_{0}-2 \sqrt{1-\hbar \omega / E_{0}} \cos \phi}
$$

Le vecteur de diffusion $Q$ est alors donné par l'angle de diffusion $\phi$ à partir du moment où les énergies incidentes et diffusées sont connues. Pour un monocristal, il faudra aussi connaître l'orientation du cristal par rapport au faisceau incident, $\psi$, comme cela sera discuté dans la section 8 . 


\section{SECTIONS EFFICACES ET FONCTIONS DE CORRELATION}

La quantité mesurée dans une expérience de diffusion inélastique avec des neutrons non polarisés (le cas des neutrons polarisés est traité par Louis-Pierre Regnault) est l'intensité du faisceau diffusé en fonction du vecteur de diffusion et de l'énergie. Cette intensité est proportionnelle à une double section différentielle, comme cela est montré dans le cours de Claude Fermon et dans les références [1-4]. Elle peut être exprimée en fonction du facteur de structure dynamique magnétique $S(\mathrm{Q}, \omega)$ par

$$
\frac{d^{2} \sigma}{d \Omega d E_{1}}=(2 p)^{2} \frac{1}{N} \frac{k_{1}}{k_{0}} S(\mathbf{Q}, \omega)
$$

où $p=0.2696 \times 10^{-12} \mathrm{~cm}$ est une constante numérique reliée à la force de l'interaction entre le neutron et les électrons non appariés. Le facteur de structure dynamique est :

$$
S(\mathbf{Q}, \omega)=\frac{1}{2 \pi \hbar} \int_{-\infty}^{\infty} \mathrm{d} t \exp (-\mathbf{i} \omega t)\left\langle\mathbf{D}_{\perp}^{\dagger} \cdot \mathbf{D}_{\perp}(t)\right\rangle
$$

où \langle\rangle représente une moyenne thermique et $\mathbf{D}_{\perp}$ est donné par

$$
\mathbf{D}_{\perp}=\hat{\mathbf{Q}} \times(\mathbf{D} \times \hat{\mathbf{Q}}) \text {. }
$$

Ici, $\hat{\mathbf{Q}}$ est le vecteur unitaire le long de $\mathbf{Q}$ et $\mathbf{D}$ est l'opérateur d'interaction magnétique de Heisenberg, qui dépendant du temps. $\mathbf{D}$ est relié à la transformée de Fourier de la densité d'aimantation totale $\mathbf{M}(\mathbf{r})$, qui inclue les moments orbitaux et de spin. Il est facile de comprendre que le neutron mesure le moment cinétique total puisque le neutron est diffusé par l'interaction de son moment magnétique avec le champ magnétique créé par les électrons non appariés et ce dernier est donné par l'aimantation totale. Dans le cas de la diffraction X magnétique, les contributions de spin et d'orbite peuvent en principe être séparées. L'équation (5) exprime que le facteur de structure est la transformée de Fourier du spectre des fluctuations magnétiques, puisqu'il corrèle la (transformée de Fourier de la) densité d'aimantation à $t=0$, $\mathbf{D} \equiv \mathbf{D}(t=0)$, avec celle à $t$ fini, $\mathbf{D}(t)$. Le terme entre crochets de l'équation (5) est une fonction de corrélation. L'interaction magnétique est telle que seules les fluctuations (ou les moments statiques) qui sont perpendiculaires au vecteur d'onde $\mathbf{Q}$ sont observées. Ceci est donné par le double produit vectoriel de l'équation (6).

Une expression plus explicite (et plus compliquée) du facteur de structure [voir le cours d'introduction par C. Fermon pour l'explication des symboles] est :

$$
\begin{aligned}
S(\mathbf{Q}, \omega) & =\sum_{\alpha \beta}\left(\delta_{\alpha \beta}-\hat{Q}_{\alpha} \hat{Q}_{\beta}\right) \sum_{\lambda_{0} \lambda_{1}} p_{\lambda_{0}}\left\langle\lambda_{0}\left|D_{\alpha}^{\dagger}\right| \lambda_{1}\right\rangle\left\langle\lambda_{1}\left|D_{\beta}\right| \lambda_{0}\right\rangle \\
& \times \delta\left(\hbar \omega+E_{\lambda_{0}}-E_{\lambda_{1}}\right)
\end{aligned}
$$

où la somme sur les coordonnées cartésiennes $\alpha$ et $\beta$ expriment (encore) que seules les composantes perpendiculaires à $\mathbf{Q}$ sont observées.

Nous avons besoin de deux relations générales en plus. La première est le principe de la balance détaillé :

$$
S(\mathbf{Q}, \omega)=\exp \left(\hbar \omega / k_{B} T\right) S(-\mathbf{Q},-\omega),
$$

qui exprime que la probabilité de créer une excitation d'énergie $\omega>0$, donnée par $S(\mathbf{Q}, \omega)$, est reliée à la probabilité d'annihiler cette même excitation, donnée par $S(\mathbf{Q},-\omega)$, à travers le facteur d'occupation des niveaux $\exp \left(\hbar \omega / k_{B} T\right.$ ), où $k_{B}$ est la constante de Boltzmann. (Pour des systèmes centrosymmetriques, le signe devant le vecteur d'onde peut être négligé). Ceci est 


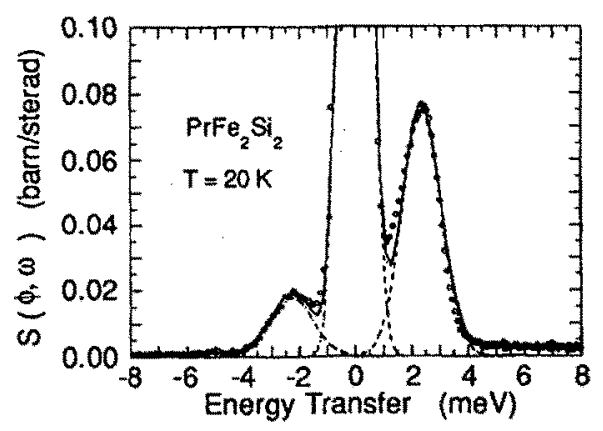

Figure 1: Une excitation de champ cristallin observée à énergie de transfert positive et négative dans $\mathrm{PrFe}_{2} \mathrm{Si}_{i_{2}}$. Le pic à énergie nulle est principalement du à la diffusion élastique incohérente. De $\mathrm{A}$. Blaise et al., J. Phys. Cond. Matter 7 (1995) 8317.

illustré sur la figure 1, où le rapport des hauteurs des pics aux énergies positives et négatives est 4, en accord avec $\exp \left(\hbar \omega / k_{B} T\right)$ pour une énergie de $2.4 \mathrm{meV}$ à une température de 20 K. L'équation (8) montre qu'à température nulle, $S(\mathbf{Q},-|\omega|)$ est nul. Cela veut dire qu'à $T=0$, les excitations peuvent seulement être créées. Ceci est attendu car il n'y a pas d'états thermiquement excités à température nulle.

Pour la diffusion magnétique, il peut être utile de décrire le système en termes de susceptibilité magnétique $\chi(\mathbf{Q}, \omega)$, dont la partie imagninaire est reliée au facteur de structure par

$$
S(\mathbf{Q}, \omega)=\frac{1}{\pi} \frac{1}{1-\exp \left(-\hbar \omega / k_{B} T\right)} \chi^{\prime \prime}(\mathbf{Q}, \omega)
$$

Comme $\chi^{\prime \prime}(\mathbf{Q}, \omega)$ est une fonction impaire, on voit facilement que l'équation (9) vérifie l'équation (8). La susceptivilité dépendant de $Q$ est donnée par

$$
\chi(\mathbf{Q})=\frac{1}{\pi} \int_{-\infty}^{\infty} \frac{\chi^{\prime \prime}(\mathbf{Q}, \omega)}{\omega} \mathrm{d} \omega
$$

qui est égale à la susceptibilité statique quand $Q \rightarrow 0$.

Dans les sections 4 et 5 , nous donnerons deux exemples d'excitations largement étudiées, la première où les énergies d'excitation sont indépendantes de $Q$ (propriétés d'un ion isolé), la seconde où les excitations sont dispersives à cause d'un couplage entre ions voisins.

\section{EXCITATIONS DE CHAMP CRISTALLIN}

Nous allons considérer un système où les moments magnétiques sont raisonablement bien localisés et où le couplage entre ceux-ci est faible. C'est le cas de composés à bases de terres rares. L'état fondamental d'un ion est donné par la règle de Hund et est caractérisé par son moment angulaire total $\mathbf{J}$, qui est la somme des moments orbitaux et de spin. Le champ électrique inhomogène créé par l'environement cristallographique sépare (au moins partiellement) le niveau fondamental, $(2 J+1)$ fois dégénéré, et des transitions entre ces états appelés états de champ cristallin peuvent être observées par diffusion de neutrons inélastique. Ces excitations sont indépendantes de $Q$ car elles sont inherentes aux propriétés d'un ion unique. 
Si les moments sont localisés et $Q$ n'est pas trop grand, l'approximation dipolaire peut être utilisée pour l'opérateur interaction magnétique,

$$
\mathbf{D}=\frac{1}{2} g f(\mathbf{Q}) \mathbf{J}
$$

où $g$ est le facteur de Landé, J le moment anglaire total et $f(\mathbf{Q})$ le facteur de forme magnétique, qui, comme pour la diffusion de rayons $X$, prend en compte l'extension spatiale de la fonction d'onde électronique localisée. Habituellement, $f(\mathbf{Q})$ decroît avec $Q$ croissant.

L'intensité de la transition induite par les neutrons entre deux niveaux de champ cristallin $\Gamma_{n}$ et $\Gamma_{m}$ dépend des éléments de matrice $\left\langle\lambda_{1}|\mathbf{D}| \lambda_{0}\right\rangle=(1 / 2) g f(Q) \times\left\langle\Gamma_{m}|J| \Gamma_{n}\right\rangle$ et de la probabilité d'occupation $p_{n}$. En utilisant l'équation (11) dans (7) et les propriétés de symétrie des éléments de la matrice de transition, on obtient:

$$
S(\mathbf{Q}, \omega)=\mathrm{e}^{-2 W}\left[\frac{g}{2} f(\mathbf{Q})\right]^{2} p_{n} \sum_{\alpha}\left(1-\hat{Q}_{\alpha}^{2}\right)\left|\left\langle\Gamma_{m}\left|J^{\alpha}\right| \Gamma_{n}\right\rangle\right|^{2} \delta\left(\hbar \omega+E_{n}-E_{m}\right),
$$

où $\exp (-2 W)$ est le facteur de Debye-Waller. La mesure à différentes valeurs de $\mathbf{Q}$ sur un monocristal permet de distinguer les transitions de champ cristallin transverses $(\alpha=x, y)$ et longitudinales $(\alpha=z)$. Cependant, les mesures des excitations de champ cristallin sont souvent faites sur des échantillons polycrystallins (poudre). Dans ce cas, l'équation (12) doit être moyennée dans l'espace de $\mathbf{Q}$, ce qui conduit à $\left(1-\hat{Q}_{\alpha}^{2}\right)=2 / 3$.

Les excitations de champ cristallin ont des énergies typiques de l'ordre de 1-80 meV, permettant d'être étudiées avec des neutrons thermiques. Un exemple de facteur de structure est donné sur la figure 1. En utilisant des neutrons de haute énergie sur des sources à spallation, des transitions entre différents $J$ ont été observées. Ces excitations sont dites intermultiplet et ont des énergies allant jusqu'à $1800 \mathrm{meV}$. Dans tous ces cas, les excitations sont essentiellement indépendantes de $Q$, car elles ne mettent en jeu qu'un seul ion. Les méthodes de temps de vol sont particulièrement bien adaptées à ces cas là.

\section{ONDES DE SPIN}

Dans beaucoup de systèmes possédant des spins localisés, les excitations de base sont des ondes de spin. Ces excitations sont créées à partir d'un état fondamental classique. Pour un ferromagnétique, cet état est l'état fondamental exact où tous les spins sont alignés dans la même direction. L'onde de spin correspond à une petite deviation transverse, comme cela est montré sur la figure 2. Dans les antiferromagnétiques, l'état fondamental classique, appelé état de Néel, correspond au plus proches voisins alignés antiferromagnétiquement. Cet état n'est pas l'état fondamental exact ni même un état propre de l'Hamiltonien. Pourtant la description d'ondes de spin est adaptée et utile en utilisant cet état fondamental. Dans les deux cas, la. déviation des spins se propage à travers le réseau, d'où le nom d'ondes de spin. Les ondes de

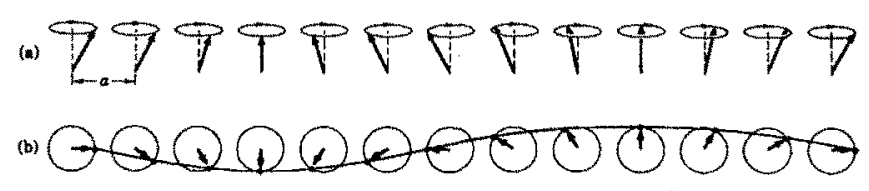

Figure 2: Vue schématique d'ondes de spin. 
spins, appelées aussi magnons, sont des excitations quantifiées (bosons) comme les phonons sont la description quantique des vibrations du réseau.

La description mathématique part de l'Hamiltonien de Heisenberg

$$
\mathcal{H}=-\sum_{i, j} J\left(\mathbf{r}_{i}-\mathbf{r}_{j}\right) \mathbf{S}_{i} \cdot \mathbf{S}_{j}
$$

où $J$ est l'intégrale d'échange qui couple différents spins $\mathbf{S}$ (où moments angulaires totaux) aux autres. Pour les plus proches voisins, $J$ est positif pour les ferromagnétiques et négatif pour les antiferromagnétiques. La relation de dispersion des ondes de spins dans un ferromagnétique de Heisenberg est donnée par

$$
\hbar \omega(\mathbf{q})=2 S[\mathcal{J}(0)-\mathcal{J}(\mathbf{q})]
$$

où $\mathcal{J}(\mathbf{q})$ est la transformée de Fourier de l'interaction d'échange $J(\mathbf{r}): \mathcal{J}(\mathbf{q})=\sum_{\mathbf{r}} J(\mathbf{r}) \exp (-\mathbf{i q} \cdot \mathbf{r})$. Pour des petites valeurs de $q$, la dispersion est quadratique en $q, \hbar \omega_{\mathrm{q}} \simeq D q^{2}$, où $D$ est appelée constante de raideur. Si le réseau est cubique et l'échange entre plus proches voisins est dominant, on trouve $D=2 J S a^{2}$. Pour un antiferromagnétique, la dispersion est en principe linéaire en $q$. En présence d'un petit terme anisotropie $g \mu_{B} H_{A} \sum_{i} S_{i}^{z}$ dans l'Hamiltonien, le spectre d'ondes de spin présente un gap de $g \mu_{B} H_{A}$ au centre de la zone de Brillouin magnétique, à partir duquel la dispersion par quadratiquement en fonction de $q$. Un exemple de dispersion d'ondes de spin est donné sur la figure 3.

Le facteur de structure d'une onde de spin peut s'écrire :

$$
\begin{aligned}
S(\mathbf{Q}, \omega) & =f^{2}(\mathbf{Q})\left(1+\hat{\mathbf{Q}}_{z}^{2}\right) \frac{1}{2} S \mathrm{e}^{-2 W_{\mathbf{Q}}} \\
& \times \sum_{\mathbf{q}, \tau}\left[\left\langle n_{\mathbf{q}}+1\right\rangle \delta(\mathbf{Q}-\tau-\mathbf{q}) \delta\left(\hbar \omega-\hbar \omega_{\mathbf{q}}\right)\right. \\
& \left.+\left\langle n_{\mathbf{q}}\right\rangle \delta(\mathbf{Q}-\tau+\mathbf{q}) \delta\left(\hbar \omega+\hbar \omega_{\mathbf{q}}\right)\right]
\end{aligned}
$$

où $\left\langle n_{\mathbf{q}}\right\rangle$ est le facteur d'occupation thermique $\left[\exp \left(\hbar \omega / k_{B} T\right)-1\right]^{-1}$. Le premier terme de la somme correspond à la création de magnons et le second à l'annihilation de magnons. Les fonctions delta assurent la conservation du moment et de l'énergie dans le processus de diffusion. Cette condition est très stricte : Pour voir une onde de spin à une énergie donnée $\omega=\omega_{\mathrm{q}}$, il faut faire la mesure à la valeur de $q$ associée.

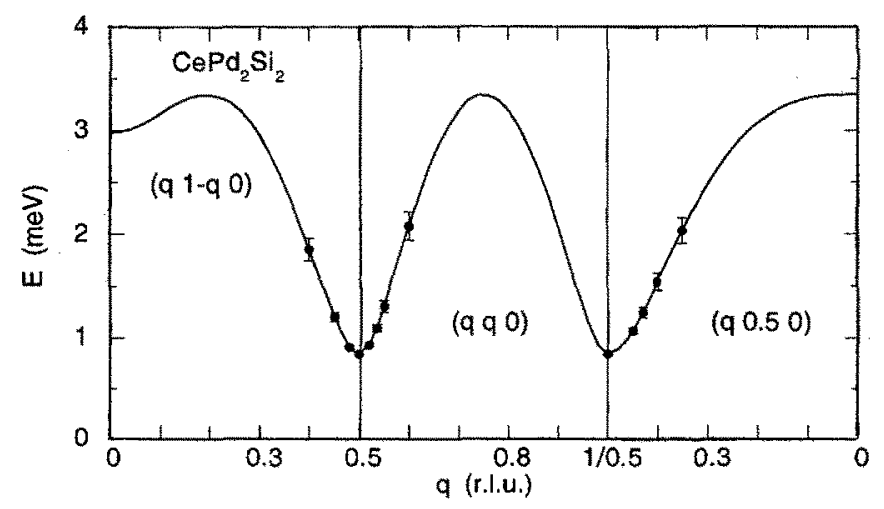

Figure 3: Dispersion d'ondes de spin pour différentes directions dans le plan de base d'un antiferromagnétique tétragonal CePd2 Siz. De N.H. van Dijk et al., Phys. Rev. B. 61 (2000) 8922. 


\section{AUTRES EXCITATIONS MAGNETIQUES}

Il existe une grande variété d'autres excitations magnétiques. Par exemple, il y a des systèmes où le champ cristallin est un bon point de départ mais où les interactions d'échange ne sont pas négligeables, entraînant une dispersion des niveaux de champ cristallin. Ce type d'excitations sont appelés excitons magnétiques. Les électrons peuvent aussi être délocalisés, comme dans le cas du magnétisme itinérant. En plus d'un continuum de type Stoner créé par les excitations électrons-trous, il peut y avoir des ondes de spin bien définies dans la phase ordonnée, dans la mesure où l'interaction d'échange est assez forte. Dans des systèmes presque ferromagnétiques, une augmentation importante des fluctuations de spin de basse énergie peut être observée. Ces excitations sont appelées paramagnons. Une revue des excitations dans des systèmes magnétiques est donnée dans [5]. Des sujets étudiés actuellement portent sur la réponse magnétique dans des systèmes d'électrons fortement corrélés comme les supraconducteurs à haute $T_{\boldsymbol{c}}$, les fermions lourds, les liquides non-Fermi ou encore les excitations dans des systèmes magnétiques quantiques de basse dimensionnalité avec des spin essentiellement localisés, où la nature quantique demande un état fondamental non classique pour une description de la dynamique.

\section{INSTRUMENTATION}

Afin de déterminer l'énergie transferée du neutron vers le système, nous avons besoin de connaître les énergies incidentes et finales (ou les vecteurs d'onde $\mathrm{k}$ ) du neutron. Il y a principalement deux méthodes utilisées (en oubliant le spin écho) : diffraction de Bragg sur un cristal analyseur (ou monochromateur) et le temps de vol. La première méthode utilise la relation entre la longueur d'onde $\lambda$ et l'angle de diffraction $\theta$ lors de la diffraction sur un monocristal de distance interrécticulaire $d$, à savoir la loi de Bragg : $\lambda=2 d \sin \theta$ ou $k=\pi /(d \sin \theta)$. En tournant le cristal analyseur (ou monochromateur), différentes énergies du neutron incident ou diffusé peuvent être choisies. La méthode du temps de vol détermine ou sélectionne l'énergie du neutron en mesurant le temps $t$ que met le neutron pour parcourir une distance $L$, c'està-dire la vitesse $v$ du neutron. Dans ce cas $k$ est donné par la relation $\hbar k / m=v=L / t$. Il faut déterminer l'énergie du neutron avant et après la diffusion. Chaque méthode peut être utilisée deux fois ou bien on peut combiner les deux techniques. Cela donne quatre possibilités pour le dessin d'un spectromètre de diffusion inelastique des neutrons et toutes sont utilisées en pratique. Dans la suite, nous allons commenter les quatre possibilités en soulignant leurs avantages respectifs.

\section{SPECTROMETRES TROIS AXES}

Le spectromètre à trois axes (TAS) est quasiment exclusivement utilisé pour déterminer les excitations dispersives (dépendantes de $Q$ ) dans des monocristaux. Des exemples typiques de TAS sont IN8 et IN14 à l'ILL, ou 1T au LLB. La configuration dans l'espace réel est montré sur la figure $4 \mathrm{a}$ et dans l'espace réciproque sur la figure $4 \mathrm{~b}$. Des monocristaux sont utilisés comme monochromateur (pour déterminer $k_{0}$ ) et comme analyseur (pour déterminer $k_{1}$ ). Un point de l'espace réciproque ( $Q$ et $\hbar \omega$ ) est mesuré à chaque fois (car il y a un seul monochromateur et un seul analyseur), ce qui est très lent. Cependant, l'avantage est de pouvoir mesurer exactement le point voulu dans l'espace réciproque, dans la mesure où il est cinématiquement accessible. En particulier, on peut mesurer le transfert d'énergie à $Q$ constant. Un tel exemple est donné sur la figure 4 b pour une énergie incidente constante (on peut aussi garder l'énergie finale constante). 
Les lignes continues donnent $k_{0}$ et $k_{1}$ pour une diffusion élastique, les lignes pointillées pour un transfert d'énergie positif. Afin de garder $\mathbf{Q}$ constant pour un $\omega$ plus grand ( $k_{1}$ plus petit), il faut changer l'angle de diffusion $\phi$ (l'angle entre $\mathbf{k}_{0}$ et $\mathbf{k}_{1}$ ) et l'orientation $(\psi)$ du cristal par rapport à $\mathbf{k}_{0}$. L'intensité est alors mesurée en fonction du transfert d'énergie. Pour chaque point du scan, quatre angles doivent être changés : l'orientation de l'échantillon $\psi$, l'angle de diffusion $\phi$, l'angle de Bragg de l'analyseur $\theta_{a}$ et l'angle du détecteur $2 \theta_{a}$. Au passage, nous pouvons noter que les spectromètres à rétrodiffusion, rarement utilisés pour les études magnétiques, sont basés sur le même principe que les TAS, mais avec une meilleure résolution en énergie et une plus mauvaise en $Q$. Les effets de résolution sont discutés dans le cours de Bruno Dorner.

\section{SPECTROMETRES PUREMENT TEMPS DE VOL}

Les spectromètres à temps de vol (TOF) sont surtout utilisés pour des échantillons polycrystallins (ou poudres), afin d'étudier des excitations qui ne sont pas (ou peu) dispersives, comme par exemple les excitations de champ cristallin discutées dans la section 4 . Cette technique est cependant de plus en plus utilisée pour des monocristaux en particulier sur des sources pulsées. Ici, nous parlerons d'abord des temps de vol "purs", où le temps de vol est utilisé pour définir les énergies incidentes et diffusées. Des exemples typiques sont IN5 (ILL), Mibemol (LLB) et MARI (ISIS). Sur une source continue, une énergie incidente est donnée par deux choppers tournant à la même vitesse mais avec différentes phases. (Un chopper est un système tournant qui ne laisse pas qu'un pulse court de neutron par tour [ou quelques-uns]). Le premier chopper produit des courts pulses de neutrons (typiquement d'une durée de quelques microsecondes). Les neutrons avec différentes vitesses arrivent sur le second chopper, placé à quelques mètres à différents temps et la phase (temps d'ouverture) du second chopper est ajusté pour laisser passer ceux avec la bonne énergie. Sur une source pulsée, le premier chopper n'est pas nécessaire, car la source elle-même produit les pulses de neutrons. En réalité, d'autres choppers sont utilisés pour réduire le bruit de fond, etc. En mesurant le temps mis pour arriver au détecteur (tous les neutrons arrivent sur l'échantillon au même moment), l'énergie finale est déterminée. Cela permet d'obtenir l'énergie de transfert.

(a)

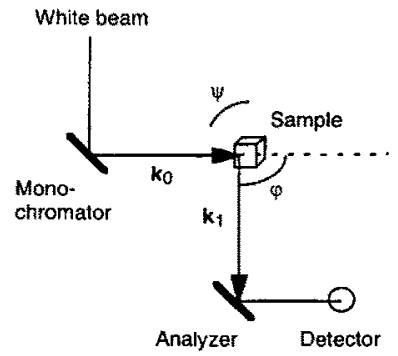

(b)

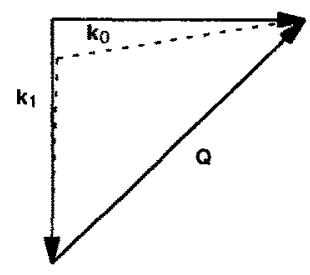

Figure 4: Spectromètre trois axes. (a) Espace réel. (b) Un scan à $\mathbf{Q}$ constant dans l'espace réciproque. Les angles $\phi$ (entre $\mathbf{k}_{0}$ et $\mathbf{k}_{1}$ ) et $\psi$ (entre $\mathbf{k}_{0}$ et une orientation crystallographique de l'échantillon) sont changés pendant un scan. 
(a)

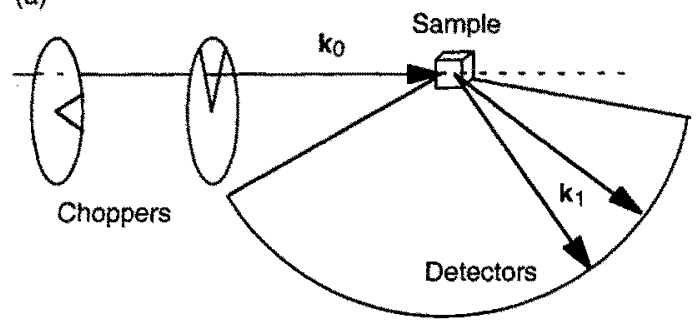

(b)

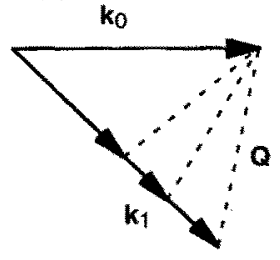

(c)

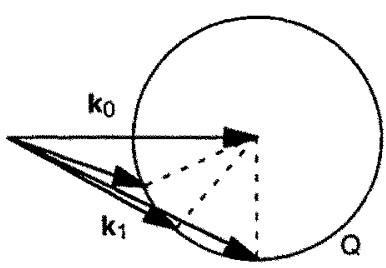

Figure 5: Spectromètre temps de vol à géométrie directe: (a) espace réel, (b) et (c) espace réciproque. Le vecteur d'onde incident $\mathbf{k}_{0}$ est fixé en amplitude et direction (par rapport au cristal). Pour un angle de diffusion fixé (b), l'amplitude et la direction de $\mathbf{Q}$ (montré par les lignes pointillées) change en fonction de l'énergie de transfert (variation de $\mathbf{k}_{1}$ ). Un scan à l'amplätude $Q$ constant peut être obtenu en combinant les informations des différents angles de diffusion, comme cela est montré en (c) lle cercle correspond à un $Q$ fixél, mais la direction de $\mathbf{Q}$ change toujours.

Le grand avantage de cette technique est que de nombreux détecteurs peuvent être utilisés simultanément et donc une large portion de l'espace réciproque est mesuré en même temps. Cela compense le faible flux incident. Pour un angle diffusé fixé, l'amplitude du vecteur de diffusion varie en fonction du transfert d'énergie [voir l'équation (3)], mais différents angles peuvent être combinés pour donner un scan à amplitude de $Q$ constant. Cela est très utile pour des échantillons polycrystallins. Cependant, comme cela est montré sur la figure 5, le scan n'est pas fait à vecteur $\mathbf{Q}$ constant car l'angle entre le faisceau incident et l'échantillon est fixé. En faisant des mesures pour des orientations cristallines différentes il est possible de tracer les relations de dispersion, mais cela coûte beaucoup en temps. Pour des systèmes de basse dimensionnalité (1D ou 2D), ce défaut peut être eliminé en alignant correctement le cristal par rapport à $\mathbf{k}_{0}$.

\section{TEMPS DE VOL HYBRIDES}

Le terme "hybride" s'applique aux instruments utilisant le temps de vol combiné à une refiexion sur un monocristal. On peut distinguer deux types :

(1) La géométrie directe: On travaille à énergie incidente constante donnée par un cristal monochromateur. L'exemple le plus connu est IN6 (ILL). Les spectromètres temps de vol purs travaillent aussi en géométrie directe car ils sont à énergie incidente constante. Leurs mode d'opération est discuté dans la section 9.

(2) La géométrie inverse : Dans ce cas, l'énergie finale est constante grâce à un réseau de 
(a)

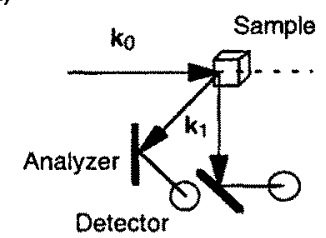

(c)

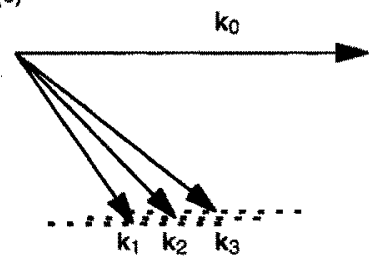

(b)

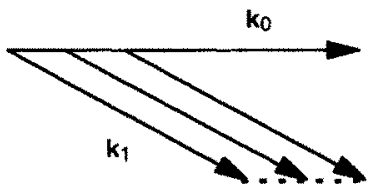

(d)

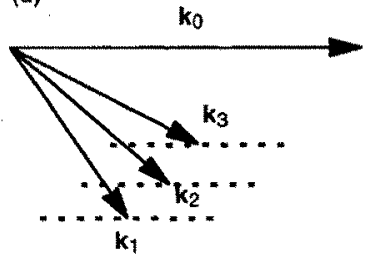

Figure 6: Spectromètre temps de vol à géométrie inverse : (a) espace réel, (b-d) espace réciproque. Le vecteur d'onde diffusé $\mathbf{k}_{1}$ est fixé en amplitude et direction par rapport à l'échantillon. Le vecteur incident est fixé en direction, mais son amplitude varie avec le temps de vol. Le vecteur de diffusion varie donc en fonction du transfert d'énergie comme cela est montré en (b). Il est alors utile de mettre un axe de haute symétrie le long de $\mathbf{k}_{0}$. (c) montre l'utilisation de différents modules cristal analyseur-détecteur, chacun avec un différent $k_{1}$ (appelés $k_{2}, k_{3}$ sur la figure), un scan à $\mathbf{Q}$ constant peut être obtenu (dans certaines limites), puisque le point $\mathbf{Q}$ correspondant $\dot{a} k_{2}$ et $\hbar \omega_{2}$, est aussi vu par les analyseurs-détecteurs numéros 1 et 3 , mais à des énergies différentes. (d) est un montage utile pour les systèmes $2 D$ avec les bâtons magnétiques le long de $\mathbf{k}_{0}$. Les différents détecteurs mesurent la dispersion perpendiculaire aux bâtons.

cristaux analyseurs. Les exemples sont PRISMA et IRIS (ISIS). Le diagramme de diffusion pour un scan en énergie en géométrie inverse est montré sur la figure 6. Dans ce cas, l'énergie incidente est déterminée par le temps de vol, ce qui implique que le faisceau incident contient une large bande de longueurs d'onde. Dans une mesure en temps de vol, $\mathbf{Q}$ change le long de $\mathbf{k}_{0}$. Si le cristal est orienté avec un axe de haute symétrie le long de $\mathbf{k}_{0}$, les relations de dispersion dans cette direction peuvent être mesurées. Si le système a une dimensionnalité plus basse, par exemple 2, la dispersion perpendiculaire à $\mathbf{k}_{0}$ peut être mesurée si les "bâtons" ("rods") magnétiques sont alignés le long de $\mathbf{k}_{0}$, en utilisant une configuration analyseurs-détecteurs différente.

\section{LE FUTUR}

Des progrès récents en diffusion inélastique de neutrons peuvent accroître fortement les performances des spectromètres existants ou futurs, et ainsi ouvrir de nouvelles et interessantes possibilités pour l'étude des excitations magnétiques. Les principaux développements sont les multidétecteurs sur les sources pulsées, les analyseurs multiples sur les trois axes (RITA), les techniques de temps de vol en spin-écho et les supermiroirs en général pour accroître le flux. $\mathrm{L}^{\prime 3} \mathrm{He}$ polarisé pourrait aussi conduire à un gain important pour les neutrons polarisés. 


\section{REMERCIEMENTS}

Je remercie Claude Fermon pour la traduction de ce cours.

\section{References}

[1] Un des meilleurs livres de départ pour la théorie de la diffusion de neutrons est écrit par G.L. Squires, Introduction to the theory of thermal neutron scattering, Cambridge University Press, 1978.

[2] Un des apports les plus complets sont les deux livres de S.W. Lovesey, Theory of neutron scattering from condensed matter, Oxford University Press, 1984. Volume 1 est général et traite surtout de la diffusion nucléaire, tandis que le volume 2 est consacré à la diffusion magnétique.

[3] Un bon mélange d'aspects théoriques et instrumentaux principalement sur la diffusion magnétique est donné par W.G. Williams, Polarized Neutrons, Oxford University Press, 1988.

[4] Chapitre 1 de D.L. Price et K. Sköld, dans Neutron Scattering, volume 23A de Methods of Experimental Physics, K. Sköld et D.L. Price ed., Academic Press, New York, 1986, est un des plus complets traitement de la diffusion nucléaire tandis que la diffusion magnétique est traitée de façon conventionnelle.

[5] Chapitre 20 de W.G. Stirling et K.A. McEwen, dans Neutron Scattering, volume 23C de Methods of Experimental Physics, K. Sköld et D.L. Price ed., Academic Press, New York, 1987, est une revue utile des excitations magnétiques. 